\title{
Neumotórax en el Recién Nacido
}

\author{
Dr.: Francisco Barrera Q. ${ }^{1}$, Dr. Rodolfo Burdach W. ${ }^{2}$, Dr. Lduardo Ferreiro A. ${ }^{3}$, \\ Dr. Eugenio Saavedra G. ${ }^{4}$, Dr. Francisco Oda C.4
}

\section{Neumothorax in the New-Born}

\begin{abstract}
Pneumothorax and pneumodiastinum must be considered in the differential diagnosis of RDS during the neonatal period. The use of assisted ventilation has increased their incidence. Symptomatic pneumothorax developed in 12 newbotn infants at the Department Neonatology, Hospital Paula Jaraquemada, during a period of two years. Seven cases had a minimal thoracotomy procedure.

Four patients died in the first three days of the disease. There were eight survivors one week after the initial symptoms, one of them died later from a secondary infection caused by Klebsiella.
\end{abstract}

El neumotórax es una causa que debe tenerse presente dentro de la etiología de] SDR del recién nacido; con una incidencia estimada en alrededor del $0.5 \%$.

Se ha insistido que la introducción de procedimientos de ventilación asistida ha aumentado el riesgo y la incidencia de neumotórax en los Servicios de Recién Nacidos ${ }^{1-2 \cdot 3}$.

El diagnóstico oportuno y el enfrentamiento de acuerdo a los actuales esquemas de tratamiento tiende a mejorar su pronóstico.

E1 presente trabajo analiza 12 casos clínicos de neumotórax en el RN y se hace una breve actualización del tema.

\section{MATERIAL Y METODO}

Nuestra casuistica corresponde a 12 recién nacidos que presentaron neumotórax sintomático, pesquisados en un lapso de 2 años, entre el $1^{\circ}$ de Enero de 1981 y el 31 de Diciembre de 1982, de un total de 2.528 recién nacidos hospitalizados por diferentes causas.

Se analizó, a través de los datos obtenidos de las fichas clínicas, los antecedentes maternos, perinatales, características del neumotórax, tratamiento y evolución de los recién nacidos.

\section{RESULTADOS}

La incidencia observada de Neumotórax en el total de recién nacidos durante el período analizado, corresponde a $0.054 \%$, en relación a RN hospi-

'Médica Servicio de Pediatría, Servicio de Neonatología y Depto. Pediatría y Cirugía Infantil. Universidad de Chile. Hospital Paula Jaraquemada.

2 Jefe Unidad Broncopulmonares. Servicio de Pediatría. Hospital Paula Jaraquemada.

2 Medico Jefe Unidad Preferencial Pediátrica. Hospital Paula Jaraquemada.

- Médico Becado Depto. Pediatría. Universidad de Chile. Hospital Paula Jaraquemada. talizados ella alcanza a $0.47 \%$. Si intentamos correlacionarlo con el total de SDR hospitalizados durante ese lapso, la incidencia observada alcanza a $1.4 \%$ (Tabla 1 ).

Tabla 1.

Incidencia de Neumotórax Sintomático en Recién Nacidas. Hospital Paula Jaraquemada. Período: 2 Años $\left({ }^{*}\right)$

\begin{tabular}{lrc} 
No de Neumotorax & \multicolumn{1}{c}{12} & $\begin{array}{c}\text { \%de } \\
\text { Neumotórax }\end{array}$ \\
No de R.N. Vivos & 22.042 & $0.054 \%$ \\
No de R.N. Hospitalizados & 2.528 & $0.47 \%$ \\
No de SDR Hospitalizados & 840 & $1.4 \%$
\end{tabular}

(*) Lnero 1981 - Dic iembre 1982.

El análisis de los antecedentes maternos, revela en 6 casos la presencia de patología del embarazo (CIE, sintomas de parto prematuro, anemia y metrorragia).

De los 12 casos, 7 eran hombres. Respecto al tipo de parto, este fue por cesárea en 8 casos y en sólo 1 hubo antecedente de forceps. 9 pacientes eran multíparas.

En 4 casos se observó antecedentes claros de sufrimiento fetal. El puntaje de Apgar en el primer minuto de vida fue superior a 7 a excepciôn de un caso en que se observó Apgar 2 al minuto y 7 a los cinco minutos, en el cual además se había pesquisado antecedentes de sufrimiento fetal. Los pesos de nacimiento variaron entre 1260 y 4290 grs. con un promedio de 2937 grs. La edad gestacional fuctuó entre 30 y 41 semanas, con un promedio de 37 semanas.

En 7 de los casos, el neumotórax fue diagnosticado antes de 24 horas de vida y en el $92 \%$ de los casos, antes de las 48 horas de vida.

La manifestación clínica que se observó con mayor frecuencia fue la dificultad respiratoria (10 
casos), siendo en 1 caso, enfisema subcutáneo cervical, la manifestación inicial, y en otro, paro respiratorio con desviación del choque de la punta.

El diagnóstico fue radiológico en 8 casos y respecto a la ubicación del Neumotórax, este fue unilateral en 8 casos, bilateral en 3 casos y neumomediastino en 1 caso. (Tabla 2). (Figs. 1 y 2).

En la serie analizada, en 2 casos existió el antecedente de uso previo de Respirador BP-200 y en otro de CPAP.

La Tabla 3 reseña algunos aspectos del tratamiento realizado en los $\mathrm{RN}$ con Neumotórax y Neumomediastino.

En todos los casos se procedió a oxigenoterapia en Cúpula con alta concentración de $\mathrm{O}_{2}$. En algunos de los casos y debido a la gravedad clínica, se administró corticoides que no influyeron mayormente en el desenlace.

La relación causal con el uso de procedimientos de ventilación asistida será motivo de otra presentación clínica. Cabe destacar sí, que las presiones respiratorias máximas utilizadas son bajas, lo que traduce un menor riesgo de Neurnotórax.

Hubo complicaciones en el $75 \%$ de los casos. A pesar de que hubo indicación precoz de antibióticos de amplio espectro, 6 tuvieron bronconeumonia o sepsis por Klebsiella, que entorpeció la evolución del neumotórax. En l caso pudo comprobarse a través de la autopsia, la existencia de una hemorragia intraventricular, agregándose otro caso de atelectasia pulmonar y un RN que fallece de insuficiencia respiratoria aguda irreversible.

Tabla 2.

Neumotórax en el Recièn Nacido. - Características Clínicas.

\begin{tabular}{|c|c|c|c|c|c|c|c|c|c|c|c|}
\hline Гако Clínico & 1 & 2 & 3 & 4 & 5 & 6 & 7 & 8 & 9 & 10 & 11 \\
\hline Sexo & F & M & $M$ & M & $M$ & $\mathbf{F}$ & M & M & $\mathbf{F}$ & $\mathbf{F}$ & $\mathbf{M}$ \\
\hline Tipo de Parto & Ces. & Ces. & Ces. & $v_{2 g}$ & Vag. & Ces. & Foreups & $\operatorname{css}$ & Cess. & Cess. & Ces. \\
\hline $\begin{array}{l}\text { Sufrimiento } \\
\text { Fetal }\end{array}$ & - & SI & - & - & - & - & SI & - & & SI & - \\
\hline Apgar (1.5') & 9 & $2-7$ & 8 & $j-5$ & ; & 9 & 7 & $8-2$ & B 7 & 7 & 8 \\
\hline $\begin{array}{l}\text { Peso } \\
\text { Nacimiento }\end{array}$ & 2.120 & 2.980 & 2.810 & 3860 & 4.290 & 3.720 & 3.720 & 1.260 & 3.090 & 1.960 & 2.310 \\
\hline $\begin{array}{l}\text { Edad al } \\
\text { Dingenóstico }\end{array}$ & 39 hts. & $23 \mathrm{hrs}$ & 22 hrs. & 40 hrs. & $2 \mathrm{hrs}$ & 70 hrs. & $35 \mathrm{mins}$ & $40 \mathrm{~min}$. & $30 \mathrm{~min}$ & $15 \mathrm{hrs}$ & $46 \mathrm{hrs}$. \\
\hline $\begin{array}{l}\text { Tipo de } \\
\text { Neumotorax }\end{array}$ & Des. & leqdo. & Dec. & Det. & Bibal. & $\begin{array}{l}\text { Neuinome } \\
\text { dest. }\end{array}$ & Bilat. & Der. & lzydo. & Der. & Bila1. \\
\hline
\end{tabular}

Tabla 3.

Tratamiento y Evolución del Neumotórax.

\begin{tabular}{|c|c|c|c|c|c|c|c|c|c|c|c|c|}
\hline Caso Clónico & 1 & 2 & 3 & 4 & 5 & 6 & 7 & 8 & 9 & 10 & 11 & 12 \\
\hline $\begin{array}{l}\text { Uso previo } \\
\text { CPAP o }\end{array}$ & & & & & & & & & & & & \\
\hline Respirador & Res.p. &.. & - & - & . & . & - & Resp. & - & - & CPAP & - \\
\hline Totacotomia & & $\begin{array}{l}\text { Subclavic. } \\
\text { defecha. }\end{array}$ & $\begin{array}{l}\text { Subclav. } \\
\text { dertechs. }\end{array}$ & - & $B_{3}$ bah & - & $\begin{array}{l}3 \text { El } \\
\text { bibt. }\end{array}$ & $\begin{array}{l}40 \mathrm{EI} \\
\text { izqdo. }\end{array}$ & $\begin{array}{c}3 \text { El } \\
\text { agdo. }\end{array}$ & - & $\begin{array}{l}40 \mathrm{El} \\
\text { izqdo. }\end{array}$ & - \\
\hline $\begin{array}{l}\text { Ducacion } \\
\text { Toracotomia }\end{array}$ & - & 35 huts. & Is hrs. & - & $S 5$ hrs. & - & 60 hrs. & $\begin{array}{l}1 \mathrm{lur} . \\
30 \mathrm{mt}\end{array}$ & 8 hIs. & - & 60 hrs. & - \\
\hline Cortisodes & Betame1. &.. & Betamet. & - & Belamet & Betam. & $\begin{array}{l}\text { Betam. } \\
\text { Cortisol }\end{array}$ &. & Cortisel & . & - & - \\
\hline Complicac. & $\begin{array}{l}\text { Higia. } \\
\text { intrav. }\end{array}$ & GRN & $\begin{array}{c}\text { BRN } \\
\text { Schock }\end{array}$ & - & $\begin{array}{l}\text { HAN } \\
\text { Hema- } \\
\text { globi- } \\
\text { nurik. }\end{array}$ & BRN & $\begin{array}{l}\text { Scosis } \\
\text { Kleths. } \\
\text { CTV }\end{array}$ & $\begin{array}{l}\text { Insut. } \\
\text { Resp. } \\
\text { Irev. }\end{array}$ & $\begin{array}{l}\text { Alekct. } \\
\text { pulmonar. }\end{array}$ & GRA & - & - \\
\hline Desenlace & $\begin{array}{l}\text { Fallece } \\
39 \text { hes. }\end{array}$ & $\begin{array}{l}\text { Alta } \\
\text { g ds. }\end{array}$ & $\begin{array}{l}\text { Fallecec } \\
58 \text { hri. }\end{array}$ & $\begin{array}{l}\text { Alta } \\
\text { 8dt. }\end{array}$ & $\begin{array}{l}\text { Alta } \\
16 \mathrm{ds} .\end{array}$ & $\begin{array}{l}\text { Alta } \\
7 \mathrm{ds} .\end{array}$ & $\begin{array}{l}\text { ralkes } \\
10 \mathrm{ds} .\end{array}$ & $\begin{array}{c}\text { Fallece } \\
5 \mathrm{hrs}\end{array}$ & $\begin{array}{l}\text { Fallece } \\
31 \text { brs. }\end{array}$ & $\begin{array}{l}\text { Alla } \\
42 \mathrm{ds} .\end{array}$ & $\begin{array}{c}\text { Alld } \\
13 \mathbf{d x} .\end{array}$ & $\begin{array}{l}\text { Alta } \\
12 \mathrm{ds} .\end{array}$ \\
\hline
\end{tabular}




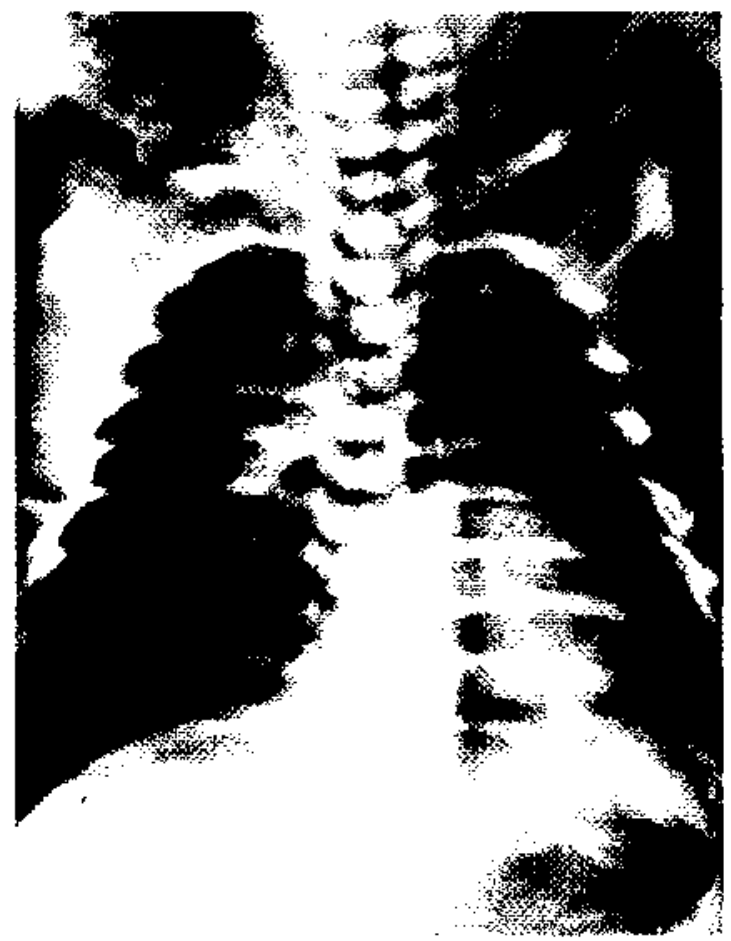

Figura l.

La radiografía Frontal de Tórax demuestra inagen de Neumotórax bilateral con enfísema subcutáneo en la Región Corvical.

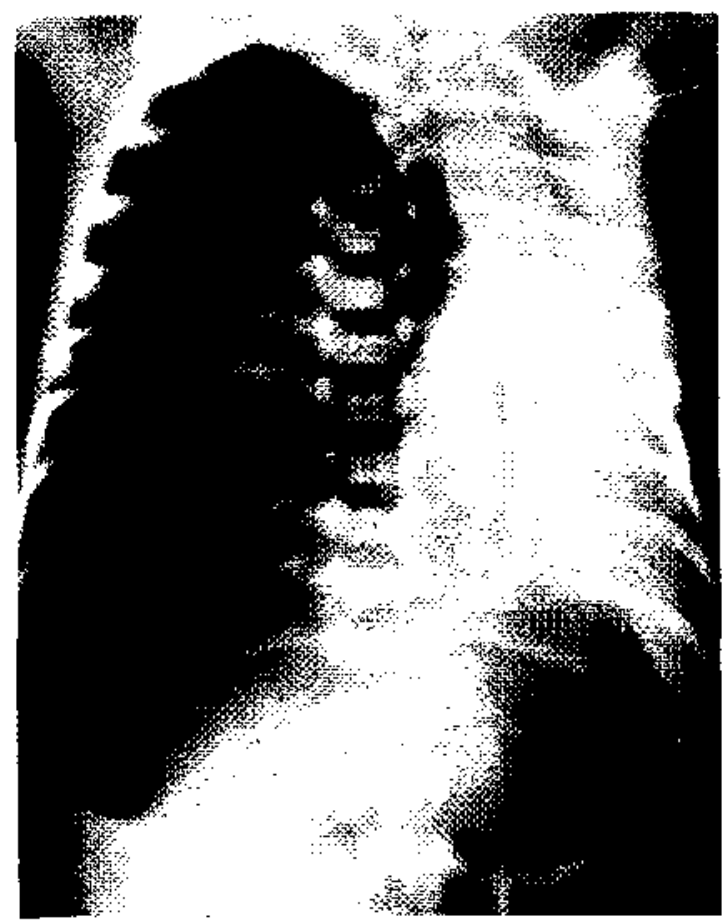

Figura 2.

Neumotórax derecho a tensión con rechazo de mediastino.
Cuatro pacientes fallecieron ( $33 \%$ ), (antes del tercer día de vida) y siete sobrevivieron y fueron dados de alta en condiciones de normalidad. Uno se complicó, después de un período de franca recuperación, falleciendo a los $\mathbf{1 0}$ dias de vida por una Septicemia causada por Klebsiella.

\section{COMENTARIO}

La presencia de 12 casos de Neumotórax de moderada a grave manifestación clínica, en nuestro Servicio de Neonatología, en un periodo de 2 antos, apoya la necesidad de tener presente este diagnóstico en determinadas circunstancias clínicas.

Publicaciones orientadas hacia la investigación sistemática de todos los recién nacidos en un servicio de neonatología, demuestran neumotórax y enfisema intersticial de grado variable en alrededor del 1 al $2 \%$ de todos los niños ${ }^{1-3-4}$. De ellos se ha planteado que el $25 \%$ pudiera dar manifestaciones clínicas de gravedad, requiriendo de hospitalización ${ }^{1}$.

En general, tiende a hablarse de neumotórax espontáneo, debido a que no se encuentran factores condicionantes o predisponentes definidos en el periodo perinatal, to que coincide con nuestra experiencia, a excepción de la hipoxia perinatal y la presencia de meconio en el momento del parto.

La existencia de sindrome de difícultad respiratoria por aspiración de un líquido amniótico con o sin meconio y las maniobras de resucitación inmediata muy enérgicas, son también factores condicionantes de neurnotórax.

El Pediatra que se enfrenta a un sindrome de dificultad respiratoria unido a estos antecedentes, debe investigar la presencia de un neumotórax. \$i bien este puede ser asintomático, las manifestaciones clínicas pueden incluir un sindrome de dificultad respiratoria de violenta aparición, progresivo, acompañado de hipersonoridad uni o bilateral, con o sir desviación del mediastino, según sea el grado de tensión y ocasionalmente puede verse enfisema subcutáneo.

Desde hace algún tiempo se ha insistido en la importancia de medir la presión arterial, debido a que antes de la aparición del neunotórax se ha encontrado un aumento significativo de ella ${ }^{6}$.

Aunque el Neunotórax puede ser sospechado clinicamente y certificado a traves de una toracocentesis, la documentación radiológica es fundamental para establecer el diagnóstico diferencial con la hernia diafragmática, quistes aéreos, enfisema lobular gigante y otras causas de sindrome de dificultad respiratoria ${ }^{2}$. También, como procedimiento auxiliar de diagnóstico, se ha planteado 
como útil, la transiuminación del tórax en cámara oscura $^{5}$.

Aunque la gran mayoría de los neumotórax son espontáneos, la secuencia básica de su fisiopatología corresponde a un aumento de la presión del aire intraalveolar con rotura de alvéolos, la que puede ser condicionada por una deficiencia de los poros de Kohn. El aire atrapado diseca el espacio intersticial y las vainas peribronquiales y perivasculares, facilitado por un mecanismo de válvula, traduciéndose inicialmente en manifestaciones de enfisema intersticial, cuya progresión puede conducir a neumomediastino, neumotórax uni o bilateral; o la combinación de ambos y aún neumopericardio ${ }^{2-3}$.

La instalación del cuadro puede ser tan violenta, que puede provocar muerte súbita, incluso sin traducción clínica ni radiológica.

Algunos accidentes graves observados guardan relación con un fenómeno de bloqueo cardiaco por el aire a gran tensión en el mediastino ${ }^{2}$.

Se ha insistido en la coexistencia de neumotórax y anomalías pulmonares o malformaciones congénitas renales o encefálicas ${ }^{2}$, lo que debe tenerse en consideración en éstos casos.

Los elementos diagnósticos fundamentales en el neumotórax son: sospecha clínica, transiluminación del tórax y toracocentesis. En algunas situaciones excepcionales, el pediatra puede no tener la oportunidad de contar con estudio radiológico, debe efectuar la toracocentesis basado únicamente en una sospecha clinica fundada.

El manejo del neumotórax y/o neumomediastino sintomático, requiere idealmente de una Unidad de Tratamiento Intensivo Neonatal, que cuente con la implementación mínima para una adecuada monjtorización desde el punto de vista cardíaco y respiratorio.

Los aspectos a considerar en el manejo del neumotórax sintomático comprenden:

Oxígeno a alta concentración (idealmente $100 \%$ en cúpula, para efectuar un lavado del Nitrógeno atrapado en el neumotórax ${ }^{3}$. Debe valorarse criticamente los riesgos de oxigenotoxicidad.

Monitoreo de la presión arterial, para pesquisar una reaparición del neumotórax.

Toracocentesis: con fines de confirmación diagnóstica y toracotomía en todo paciente portador de un neumotórax sintomática con agravamiento progresivo o que no dé lugar a otro tipo de medidas terapéuticas.

La toracocentesis con fines diagnósticos puede efectuarse con una aguja mariposa, cuya sonda distal puede ser sumergida en agua para observar las burbujas características de un neumotórax a tensión.

Existen discusiones acerca del sitio ideal para la toracotomia minima. Sin embargo hay consenso en que la taracotomia debe ser anterior debido a que el aire atrapado tiende a desplazarse hacia la parte anterior del tórax. Por otra parte, las inserciones laterales, tienden a desplazar la sonda hacia atrás. Publicaciones recientes sugieren efectuar ba toracotomía en el primer a tercer espacio intercostal por dentro de la línea medio clavicular o cuarto a sexto espacio intercostal por delante de la línea axilar anterior ${ }^{7-8}$. (Fig. 3).
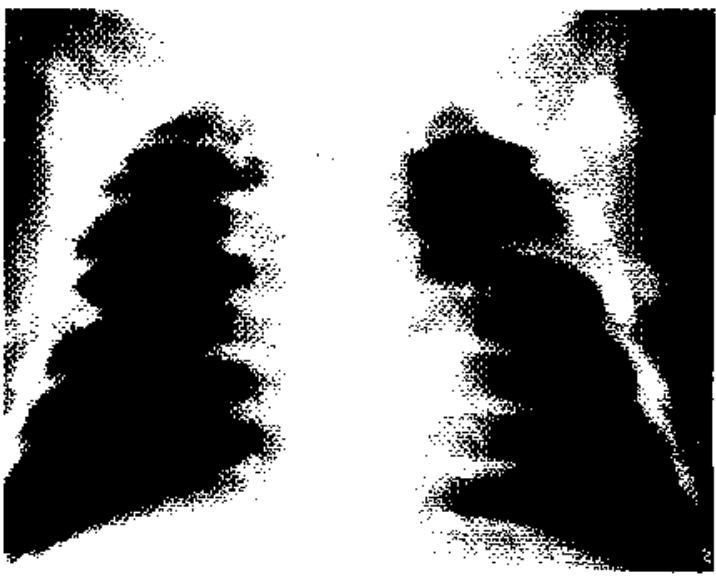

Figura 3.

Toracotom ia Bilateral en Neumotórax Grave.

Por otra parte, cuando se use sonda deberá emplearse por el menor tiempo posible, aconsejándose una permanencia de alrededor de 36 horas $^{7-8}$.

Se recomienda además el uso de antibióticos de amplio espectro en todo paciente sometido a ella.

Es posible que la incidencia de neumotórax y/o neumomediastino en los distintos centros hospitalarios varíe de acuerdo a la existencia de procedimientos de ventilación asistida y los criterios con que ellos sean utilizados, sobre todo en lo referente a presión inspiratoria máxima y ventilación mandat oria intermitente.

La mortalidad observada en neumotórax es variable, habiéndose comunicado cifras que oscilan alrededor del $30 \% \%^{-3}$. Su pronóstico estaría influenciado por la existencia de prematuridad, sufrimiento fetal, dificultad respiratoria, anomalías congénitas asociadas y maniobras de resucitación. Tambiên se ha comunicado alta mortalidad cuando se ha reconocido el enfisema intersticial. La existencia de membrana hialina, ensombrece el pronóstico de un neumotórax.

La presencia de estos casos de neumotórax en nuestra Unidad de Neonatología nos ha inducido a revisar esta patologia con el fin de actualizar su manejo. 


\section{RESUMEN}

El Neumotórax y el Neumomediastino deben tenerse presente en la etiología del SDR del Recièn Nacido. Se ha insistido en que la introducción de procedimientos de ventilación asistida ha aumentado su riesgo e incidencia. Se analizan 12 casos clínicos de Neumotórax sintomático pesquisados en el Servicio de Neonatalogía del Hospital Paula Jaraquemada en el lapso de 2 años. En el análisis destaca la falta de antecedentes predisponentes definidos. En 7 casos se efectuó toracotomía mínima. Se observó una sobrevida a la semana de vida de 8 casos $(66 \%)$. Se comenta el manejo actual del Neumotórax, a fin de mejorar sus expectativas de vida.

\section{REFERENCIAS}

1 Wesenberg, $R$. Tensión Pneumothorax and Air-block
Syndrome. The Newborn Chest. Harper and Row Ed, U.S.A. $163-170,1973$.

2 Gerbeaux, J. Patología Respiratoria en el Niño. Neumo tórax; Neumonediastino. Segunda Edición. Barcelona. Salvat. Págs. $511-514,1977$.

${ }^{3}$ Monin, $P$. Pneumothorax. Clinics in Perinatology. 5(2): $355,1978$.

4 Klaus M. y Faranoff A. Asistencia del Recién Nacido de alto riesgo. Buenos Aires, Editorial Panamericana. 1975. págs. 535 .

5 Gotrschalk, $S$. et Al. The use of a penlight as an aid in diagnosing pneumotharax. Clin. Ped. 19: 725, 1980.

${ }^{6}$ Fox, W., ef at. Pulmonary physiotherapy in Neonates. Phy siologic changes and respiratory management J. Ped. 92: 977, 1978.

? Allen, $R$. et al. Effectiveness of chest tube evacuation of pneumothorax in neonates. J. Ped. 99: 629, 1981.

9 Yung, A. et al. Pulmonary hemorrhage secondary to chest tube placement for pneumothorax in neonates. Clin. Ped. 19: 624, 1980.

${ }^{9}$ Schaffer, A. Hiperinflated lung; Lobar emphysema; Pneumothorax; Pneumomediastinum. Diseases of the Newborn. Quinta edición. Barcelona. Salvat. 1977. 